\title{
High Frequency Oscillating Ventilation (HFOV) in Severe Acute Respiratory Distress Syndrome (ARDS): A Case Report.
}

\author{
Azrina $M R^{a}$, Basri $M N^{a}$, Abdul Hadi $M^{a}$, Fahmi $M L^{a}$, Ariff $O^{a}$ and Asmarawati $M Y^{b}$ \\ a Department of Anaesthesiology and Intensive Care, International Islamic University Malaysia, \\ ${ }^{b}$ Department of Anaesthesiology, Hospital Tengku Ampuan Afzan Kuantan
}

\begin{abstract}
High frequency oscillating ventilation (HFOV) provides a rescue therapy for patients with refractory hypoxaemia in severe acute respiratory distress syndrome (ARDS). HFOV utilizes high mean airway pressures to maintain an open lung and low tidal volumes at a high frequency that allows for adequate ventilation while at the same time preventing alveolar overdistension. This seems to be an ideal lung protective ventilation strategies to prevent ventilator-induced lung injury (VILI)2. We reported a case of severe extrapulmonary ARDS secondary to acute lymphoblastic leukaemia responding to the use of HFOV.
\end{abstract}

KEYWORDS: High frequency oscillating ventilation, Acute respiratory distress syndrome

\section{INTRODUCTION}

The use of high frequency oscillating ventilation (HFOV) has been reported to be successful as a rescue therapy in the refractory hypoxaemia in acute respiratory distress (ARDS) patients. ${ }^{1}$ It utilizes the high mean airway pressures to maintain an open lung and low tidal volumes at a high frequency that allow for adequate ventilation while at the same time preventing alveolar overdistension. This seems to be an ideal lung protective ventilation strategy to prevent ventilator-induced lung injury (VILI). ${ }^{2}$

A review on published studies has shown that HFOV seems to be safe and effective for adults with severe ARDS patients who have failed conventional ventilation. ${ }^{3}$ In our setting, we first used HFOV in 2007 using 3100B ventilator in ARDS patients admitted to our ICU. We reported a case of severe ARDS in whom we initiated HFOV as a rescue therapy. The definition of ARDS follows the American-European Consensus definitions of acute lung injury (ALI) and ARDS. ${ }^{4}$

\section{CASE REPORT}

A 17 years old boy presented with 1 week history of periorbital oedema, 3 weeks history of gum hypertro-

Corresponding author;

Dr. Azrina Md Ralib

Department of Anaesthesiology and Intensive Care International Islamic University Malaysia

e-mail:drazrina@gmail.com phy and bleeding, and abdominal bloatedness. There was no previous medical history of note. On examination, he was alert and conscious, comfortable and not tachypnoeic. His vital sign was as follow, blood pressure of $130 / 80$, heart rate of 130 per minute and temperature of $38.5^{\circ} \mathrm{C}$ to $39^{\circ} \mathrm{C}$. There were signs of fluid retention with periorbital swelling and pitting oedema on both legs. He was also clinically pallid. His abdomen was soft but distended with ascites and enlarged liver.

Initial investigations revealed severe pancytopenia with haemoglobin of $9.6 \mathrm{~g} / \mathrm{dl}$, platelet count of $28 \mathrm{x}$ 103 and white cell count of $2 .{ }^{6}$. Ultrasound and CT scan of abdomen showed moderate ascites with enlarged liver and left pleural effusion. Blood products were transfused in view of persistent thrombocytopenia and anaemia. Intravenous antibiotic cefepime and metronidazole were started in view of possible intraabdominal infection. However, subsequently the patient became more lethargic. He also complained of becoming tachypnoea which later improve after peritoneal tapping. Peritoneal tapping drained 1 litre of fluid which was turbid and haemorrhagic and its biochemistry showed exudates pattern.

Bone marrow aspiration analysis was compatible with acute lymphoblastic leukaemia ALL (Stage L3). Intravenous antibiotics were later changed to meropenem and fluconazole in view of his worsening condition and possible fungal infection in neutropenic patient. However, his condition deteriorated and he became increasingly tachypnoeic. He later developed type 1 respiratory failure with severe metabolic acidosis. He was then intubated and ventilated in the intensive care unit (ICU). He also developed acute renal failure with blood urea of $51.7 \mathrm{mmol} / \mathrm{l}$ and serum creatinine of $243 \mu \mathrm{mol} / \mathrm{l}$. Continuous renal replacement therapy (CRRT) was later started. 
In ICU, he required a high ventilator setting. His oxygenation level was poor despite on high ventilator setting. He was initially ventilated on SIMV rate 12 , tidal volume of $350 \mathrm{ml}$ with PEEP of 10 and FiO2 of 1.0. However the oxygenation was still poor with partial pressure of arterial oxygen ( $\mathrm{PaO2}$ ) of only 39 and pulse oximetry (SpO2) ranging from 70 to $80 \%$.

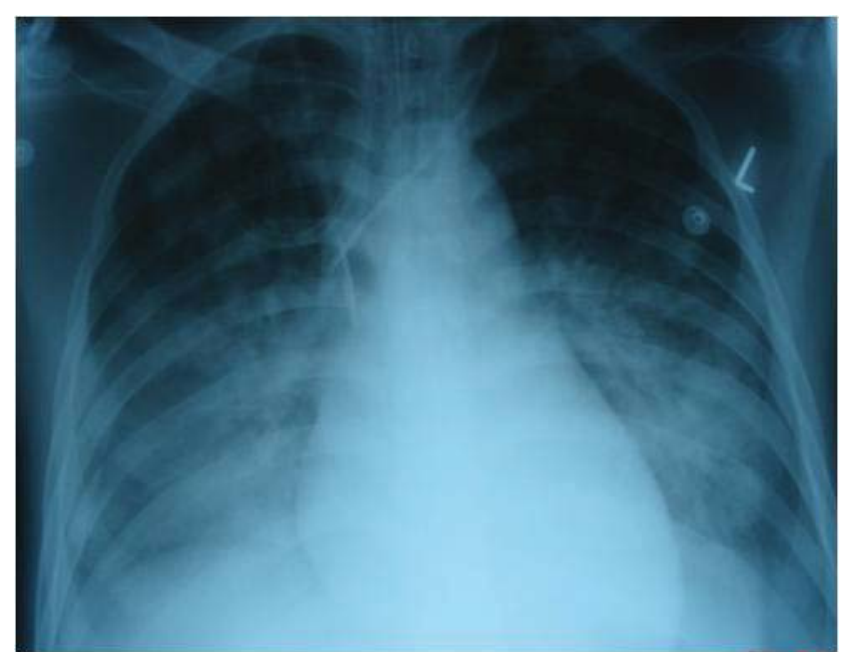

Figure 1. Bilateral infiltrates consistent with ARDS feature

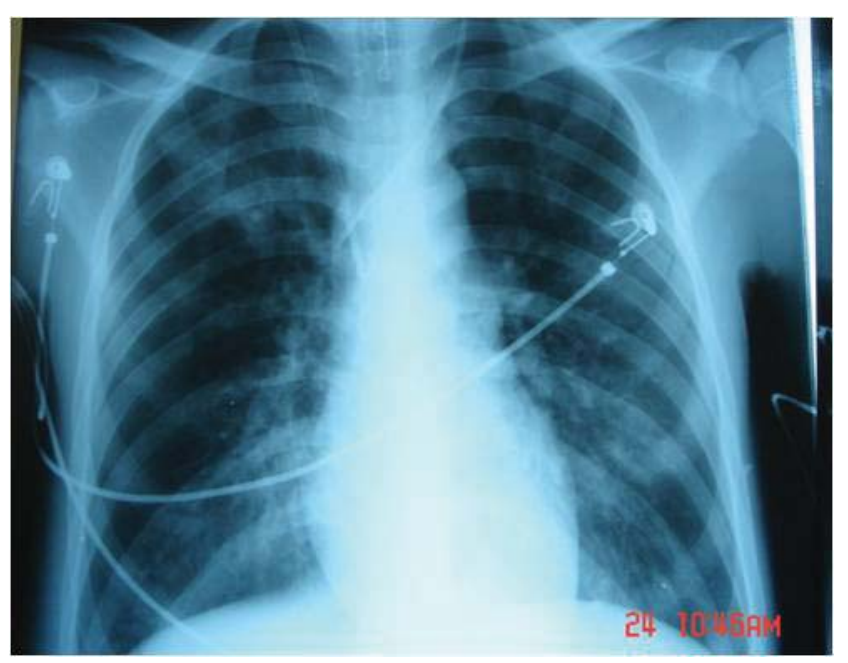

Figure 2. Improved Chest x ray

Repeated chest x-ray showed bilateral infiltrates consistent with ARDS feature. A diagnosis of ARDS secondary to acute lymphoblastic leukaemia was made based on the definition by the American-European Consensus. ${ }^{4} \mathrm{~A}$ decision to start on HFOV was made. ${ }^{5}$ He was then connected to HFOV with the setting of FiO2 1.0, mean airway pressure of $30 \mathrm{cmH}^{\circ}$, amplitude of 85 , inspiratory time of $33 \%$ and frequency of $5.5 \mathrm{~Hz}$. Paralysis and sedation were started. The aim was to maintain pulse oximetry (SpO2) between $88 \%$ to $90 \%$. Following that his $\mathrm{SpO} 2$ improved markedly to $94 \%$. Repeated arterial blood gases (ABG) showed marked improvement in oxygenation with of $\mathrm{PaO} 2$ of 87. Over the night, we were able to wean off the ventilator setting to $\mathrm{FiO} 2$ of 0.4 .

The following day, he showed much improvement in ventilation and oxygenation. HFOV was later changed to conventional ventilation after 21 hours. He was later extubated. Once his condition stabilized, chemotherapy was then started. Repeated chest $x$-ray showed a dramatic improvement.

Once his condition was stabilized, he was transferred out from ICU. His white cell count improved to 5.9 and platelet count to 175 . He was later referred to Hospital Ampang for further management of his leukaemia.

\section{DISCUSSION}

The patient developed extrapulmonary ARDS secondary to acute lymphoblastic leukaemia. ${ }^{3,6} \mathrm{His}$ baseline oxygenation was very poor with hypoxaemic index (PF ratio) of 39 and oxygenation index of 70 . Hypoxaemic index ( $\mathrm{PF}$ ratio) is derived from the ratio of $\mathrm{PaO} 2$ over FiO2. Acute lung injury is defined as PF ratio less than 300 and ARDS with PF ratio less than $200 .{ }^{4}$ Oxygenation index is used to assess the intensity of ventilatory support required to maintain oxygenation. It was derived from the following formula;

Figure 3. Oxygenation Index Formula

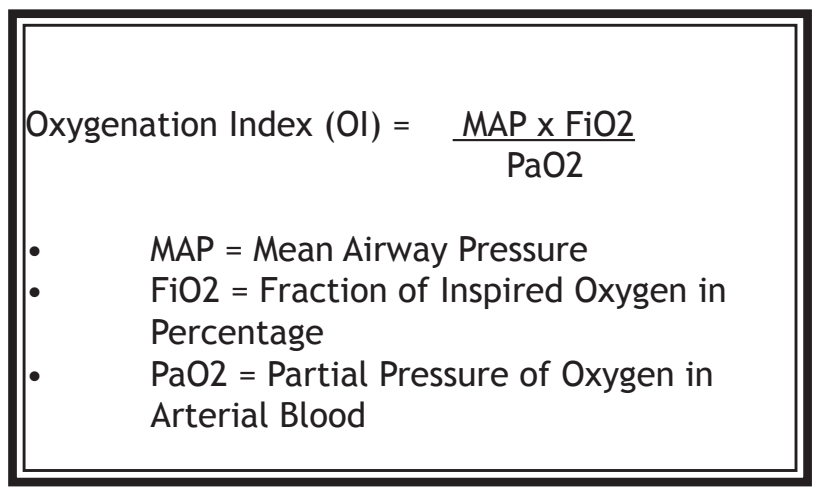

Since his oxygenation was very poor, we initiated HFOV after 1 hour of conventional ventilation. We followed a protocol on Clinical Management Strategies for Adult Patients by Stephen Derdak and also a protocol from a round-table discussion. ${ }^{5,7}$ The patient's oxygenation improved dramatically within an hour of starting HFOV and it continued to improve within the first 24 hours. These were shown in the following chart on oxygenation index and hypoxaemic index.

The use of HFOV has been shown to be effective as a rescue therapy in our patient. This is in line with other studies that showed HFOV has consistently improved oxygenation. ${ }^{8,9,10,11}$ Complications associated with the use of HFOV are usually related barotrauma (pneumothorax) and haemodynamic compromise ${ }^{1}$. Pneumothorax rate was reported at $6.3 \%$ to $22 \%$ in other studies. ${ }^{8,10}$ The higher mean airway pressure utilized during HFOV could impede venous return and lead to hypotension. However, this complication seemed to be uncommon, and only one study documented significant hypotension after the initiation of HFOV. ${ }^{11}$ Fortunately our patient had none of the above complication. 


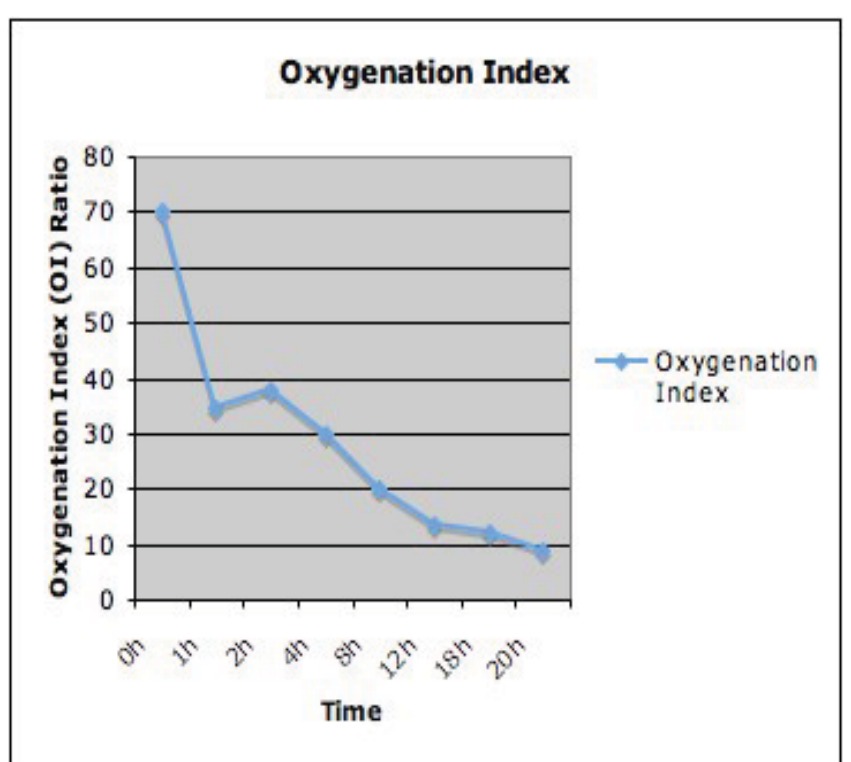

Figure 4. Oxygenation Index reduced within hours of HFOV initiation

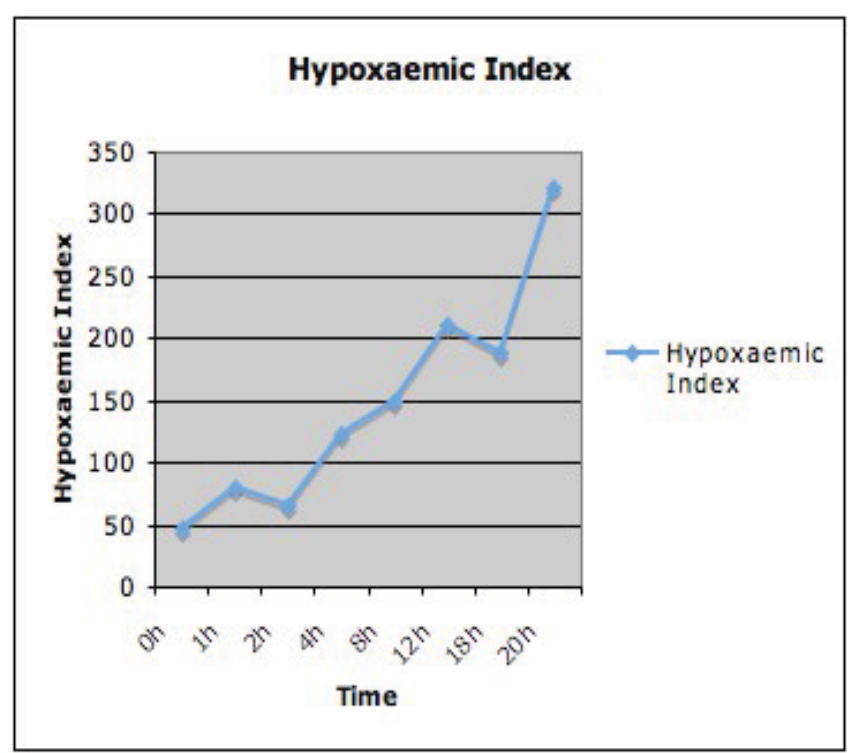

Figure 5. Hypoxaemic index increased within hours of starting HFOV

Type of ARDS in our patient was classified under extrapulmonary ARDS based on Pelosi et al. ${ }^{6}$ This is in consistent with a study by Pach et al that showed significantly higher hypoxaemic index (PF ratio) six hours after HFOV in extrapulmonary ARDS compared to pulmonary ARDS. ${ }^{12}$ This is because extrapulmonary ARDS has a lower pulmonary and chest wall compliance compared to pulmonary ARDS, hence has better recruitment of lung and improvement in oxygenation.

\section{CONCLUSION}

Our experience in using HFOV in severe ARDS in this case has been very encouraging. Complications associated with its use are also low and manageable. This is in accordance with other published data. HFOV provides a life-saving option in refractory hypoxaemia in severe ARDS.

\section{REFERENCES}

1. Rosai J, Sabin LH, Scully RE, Young RH, Clement PB. Tumors of the ovary, maldeveloped gonads, fallopian tube, and broad ligament. Atlas Tumor Pathol 1998; 160-161

2. Gruppos Italiano per lo Studio Dell'Endometriosi. Prevalence and anatomical distribution of endometriosis in women with selected gynecological condition: results from a multicentric Italian study. Human Reprod 1994; 9:1158

3. Khoo JJ, Ismail MS, Tiu CC. Endometriosis of the appendix presenting as acute appendicitis. Singapore Med J 2004; 45:435-6

4. Sait $\mathrm{KH}$. Massive ascites as a presentation in a young woman with endometriosis: a case report. Fertil Steril 2008; 90:2015

5. Stock RJ. Postsalpingectomy endometriosis: a reassessment. Obstet Gynecol 1982; 60:560-70

6. Heylen SM, Brosens IA, Puttemans PJ. Clinical value and cumulative pregnancy rates following rigid salpingoscopy during laparoscopy for infertility. Hum Reprod 1995; 10:2913-6

7. Bowman MC, Cooke ID. Comparison of fallopian tube intraluminal pathology as assessed by salpingoscopy with pelvic adhesions. Fertil Steril 1994; 61:464-9

8. Strandell A, Waldenström U, Nilsson L, Hamberger L. Hydrosalpinx reduces in vitro fertilization/embryo transfer rates. Human Reprod 1994; 9:861-3

9. Gardner KD, Weissman A, Howles MC, Shoham $Z$ eds. 3rd ed. Textbook of assisted reproductive techniques: Laboratory and clinical perspectives. New York: Informa, 2009:791-803 
